\title{
Nonlinear Compton scattering and electron acceleration in interfering laser beams
}

\author{
A. Ts. Amatuni \\ Yerevan Physics Institute, Alikhanian Brothers St. 2, Yerevan 375036, Republic of Armenia
}

I. V. Pogorelsky

Brookhaven National Laboratory, 725 C, Upton, New York 11973

(Received 1 April 1998; published 30 July 1998)

\begin{abstract}
The exact solution of the classical nonlinear equation of motion for a relativistic electron in the field of two electromagnetic (EM) waves is obtained. For the particular case of the linearly polarized standing EM wave in the planar optical cavity, intensity of the nonlinear Compton scattering, the time of flight, and the momentum variation after the relativistic electron passes along the cavity axis are calculated in weak and strong field limits. These effects depend on the initial phase of the EM wave at the electron entrance into the cavity and can be used for producing, diagnostics, and acceleration of relativistic electron (positron) microbunches. [S1098-4402(98)00007-X]
\end{abstract}

PACS numbers: 13.60.Fz, 03.40.Kf, 03.65.Ge, 14.60.Cd

\section{INTRODUCTION}

The theory of Compton effect in the interfering electromagnetic (EM) waves, in particular, in two counterpropagating plane waves, has been addressed previously to describe the Kapitza-Dirac effect [1,2], Compton lasers $[3,4]$, and inverse Compton laser acceleration [2,5]. The physical principle of the nanometer-resolution Shintake electron beam profile monitor [6-9] is also based on the understanding of Compton effect in a standing EM wave. The vacuum beat wave laser accelerator concept [10] relies on the ponderomotive acceleration resulting from the beat wave produced by the interaction of two copropagating laser beams.

Temporal diagnostics of ultrafine electron microbunches sized to a portion of the laser wavelength is another potential application for intense standing EM waves. Production and reliable characterization of such microbunches are essential for development of far-field and near-field laser accelerator schemes (see, for example, review paper [11]) into practically meaningful monochromatic electron (positron) accelerators. The example of such a scheme is the staged electron laser acceleration experiment (STELLA) at the Brookhaven Accelerator Test Facility (ATF) [12]. In this experiment, a regular train of the $1 \mu \mathrm{m}$ thick electron microbunches grouped by the inverse free electron laser method to the period equal to the $\mathrm{CO}_{2}$ laser wavelength, $\lambda=10 \mu \mathrm{m}$, is phased to the inverse Cherenkov laser acceleration stage driven by the same $\mathrm{CO}_{2}$ laser. Observation of Compton scattered radiation from the interaction of periodically grouped electrons with a standing EM wave, produced by two counterpropagating $\mathrm{CO}_{2}$ laser beams, may permit a direct assessment of the microbunch quality. The inverse process may also provide an alternative mechanism to generate microbunches starting with a quasicontinuous electron pulse.
Theoretical studies of Compton effect in two interfering EM waves, comprehensively reviewed by Fedorov [2], generally capitalize on various aspects of the perturbation theory or other approximate approaches. As long as we are interested in processes nonlinear to the field, the approach based on an exact solution of equations of motion is the most appropriate. The exact solutions of classical equations of the electron motion in a single plane EM wave have been obtained by many authors using different methods (see [13-17] and references therein). A natural extension of this approach is to apply the same methods to the case of two (or more) interfering plane waves.

In the present work, this intent is partially accomplished using the approach developed in $[14,15]$. The exact solution of classical nonlinear equations of electron motion in the field of two plane EM waves with different frequencies and the same linear polarization is found in Sec. II. It is shown that the exact solution exists also in the case of collinear (or anticollinear) plane waves linearly polarized in arbitrary directions.

The particular case of a standing wave is considered in Sec. III in the weak field and strong field approximations. We calculate the time of flight for the electron passing through the radiation filled plane-parallel optical resonator and show that this parameter depends upon the phase of the standing EM wave at the moments when the electron enters the cavity.

In Sec. IV, we calculate the phase dependent intensity radiated due to Compton scattering when the relativistic electron passes along the axis of the plane-parallel optical cavity.

Propagating along the axis of the standing EM wave, an electron can lose its energy via Compton scattering or acquire it in the inverse process. Energy gain is proportional to the initial electron energy as it is typical for the ponderomotive acceleration processes $[10,16,17]$. 
In Sec. V, we demonstrate that electron acceleration depends upon the standing wave phase as well.

\section{EXACT SOLUTION OF BASIC NONLINEAR EQUATION}

Following the approach outlined in [14,15], consider a classical equation of the electron motion in EM field

$$
\frac{d \pi_{\mu}}{d \tau}=\frac{e}{m} F_{\mu \nu} \pi_{\nu}
$$

where $\pi_{\mu}$ is the four-dimensional vector of the electron energy momentum $\pi_{\mu}(\varepsilon, \vec{p})$ equal to $\pi_{\mu}^{2}=\varepsilon^{2}-p^{2}, \tau$ is time in the relativistic frame of the electron (local or proper time), and $F_{\mu \nu}$ is a tensor of an EM field

$$
F_{\mu \nu}=\frac{\partial A_{\nu}}{\partial x_{\mu}}-\frac{\partial A_{\mu}}{\partial x_{\nu}} .
$$

The vector potential $A_{\nu}$ is

$$
A_{\nu}=a_{\nu}^{(1)}\left(\zeta_{1}\right)+a_{\nu}^{(2)}\left(\zeta_{2}\right),
$$

where $a_{\nu}^{(1)}\left(\zeta_{1}\right), a_{\nu}^{(2)}\left(\zeta_{2}\right)$ are vector potentials of two linearly polarized plane waves with frequencies $\omega_{1}$ and $\omega_{2}$, $\zeta_{1}=k_{\mu}^{(1)} x_{\mu}$ and $\zeta_{2}=k_{\mu}^{(2)} x_{\mu}$ are the phases of corresponding plane waves, and $k_{\mu}^{(1)}\left(\omega_{1}, \vec{\omega}_{1}\right)$ and $k_{\mu}^{(2)}\left(\omega_{2}, \vec{\omega}_{2}\right)$ are four-dimensional wave vectors. In Eqs. (1) and (2) and below the system of units with $c=1, h=1$ is used.

A four-dimensional electron vector $x_{\mu}$ is

$$
x_{\mu}(\tau)=\frac{1}{m} \int_{\tau_{i}}^{\tau} \pi_{\mu}(\tau) d \tau+x_{\mu}\left(\tau_{i}\right)
$$

After introducing $p_{\mu}$, the four-vector of the electron initial momentum before the electron enters the EM field, we can search a solution of Eqs. (1)-(4) in the form

$$
\begin{aligned}
\pi_{\mu}(\tau)= & p_{\mu}-e\left[a_{\mu}^{(1)}\left(\zeta_{1}\right)+a_{\mu}^{(2)}\left(\zeta_{2}\right)\right] \\
& +k_{\mu}^{(1)} f_{1}(\tau)+k_{\mu}^{(2)} f_{2}(\tau),
\end{aligned}
$$

which is a linear decomposition over four-vectors $p_{\mu}, a_{\mu}^{(1)}, a_{\mu}^{(2)}, k_{\mu}^{(1)}, k_{\mu}^{(2)}$.

From Eqs. (4) and (5), the phases of the plane waves are

$$
\begin{aligned}
d \zeta_{1} & =d\left(k_{\mu}^{(1)} x_{\mu}\right) \\
& =\frac{1}{m}\left[k^{(1)} p-e\left(k^{(2)} a^{(1)}\right)+k^{(1)} k^{(2)} f_{2}\right] d \tau, \\
d \zeta_{2} & =d\left(k_{\mu}^{(2)} x_{\mu}\right) \\
& =\frac{1}{m}\left[k^{(2)} p-e\left(k^{(2)} a^{(1)}\right)+k^{(1)} k^{(2)} f_{2}\right] d \tau .
\end{aligned}
$$

The scalar products of two four-vectors, e.g., $k_{\mu}^{(1)} k_{\mu}^{(2)}$ in Eq. (6) and later, are denoted as $k^{(1)} k^{(2)}$ or $\left(k^{(1)} k^{(2)}\right)$. In Eq. (6), the conditions $k_{\mu}^{(1)^{2}}=0, k_{\mu}^{(2)^{2}}=0, k_{\mu}^{(1)} a_{\mu}^{(1)}=0$, $k_{\mu}^{(2)} a_{\mu}^{(2)}=0$ are used. Substituting Eq. (5) in Eq. (1) and using Eqs. (2), (3), and (6), the following equations for $f_{1}$ and $f_{2}$ can be obtained:

$$
\begin{aligned}
& \frac{d f_{1}}{d \tau}=\frac{e}{m} \frac{d}{d \zeta_{1}}\left[\left(a^{(1)} p\right)-\frac{e}{2}\left(a^{(1)}\right)^{2}\right]-\frac{e^{2}}{m}\left(a^{(2)} \frac{d a^{(1)}}{d \zeta_{1}}\right)+\frac{e}{m} f_{2} \frac{d}{d \zeta_{1}}\left(k^{(2)} a^{(1)}\right), \\
& \frac{d f_{2}}{d \tau}=\frac{e}{m} \frac{d}{d \zeta_{2}}\left[\left(a^{(2)} p\right)-\frac{e}{2}\left(a^{(2)}\right)^{2}\right]-\frac{e^{2}}{m}\left(a^{(1)} \frac{d a^{(2)}}{d \zeta_{2}}\right)+\frac{e}{m} f_{1} \frac{d}{d \zeta_{2}}\left(k^{(1)} a^{(2)}\right),
\end{aligned}
$$

where $d \zeta_{1}$ and $d \zeta_{2}$ are expressed by Eq. (6) through the local time differential $d \tau$. The nonlinear system of Eq. (7) can be solved exactly when additional conditions on the transverse EM field are fulfilled,

$$
\left(k^{(1)} a^{(2)}\right)=0, \quad\left(k^{(2)} a^{(1)}\right)=0 .
$$

The conditions in Eq. (8) are valid when both plane waves have the same linear polarization or are collinear (or anticollinear) and have an arbitrary linear polarization. Below, we consider linearly polarized waves that satisfy general transverse conditions expressed by Eq. (8). The same technique can also be used for circularly polarized waves.

Using Eqs. (6) and (7), the following nonlinear system of equations for $f_{1}(\tau)$ and $f_{2}(\tau)$ is obtained:

$$
\begin{aligned}
& {\left[\left(k^{(1)} p\right)+\left(k^{(1)} k^{(2)}\right) f_{2}\right] \frac{d f_{1}}{d \tau}=e \frac{d}{d \tau}\left[\left(a^{(1)} p\right)-\frac{e}{2}\left(a^{(1)}\right)^{2}\right]-e^{2} a^{(2)} \frac{d a^{(1)}}{d \tau},} \\
& {\left[\left(k^{(2)} p\right)+\left(k^{(1)} k^{(2)}\right) f_{1}\right] \frac{d f_{2}}{d \tau}=e \frac{d}{d \tau}\left[\left(a^{(2)} p\right)-\frac{e}{2}\left(a^{(2)}\right)^{2}\right]-e^{2} a^{(1)} \frac{d a^{(2)}}{d \tau} .}
\end{aligned}
$$

The first integral of Eq. (9) can be found after summing the two parts of Eq. (9). Then, it is possible to express $f_{2}$ through $f_{1}$ and integrate the obtained equation for $f_{1}$. The integration constants can be found from the conditions $f_{1}=0, f_{2}=0$ at the initial moment $\tau=\tau_{i}$ when the electron with the initial momentum $p_{\mu}$ enters the region occupied by the EM field. Finally, we obtain the following exact solutions:

$$
f_{1}=\frac{\left(k^{(2)} p\right)}{k^{(1)} k^{(2)}}\left[\exp \left(\int_{\tau_{i}}^{\tau} F_{1} d \tau\right)-1\right], \quad f_{2}=\frac{\left(k^{(1)} p\right)}{k^{(1)} k^{(2)}}\left[\exp \left(\int_{\tau_{i}}^{\tau} F_{2} d \tau\right)-1\right],
$$


where quantities $F_{1}$ and $F_{2}$ are

$$
\begin{aligned}
& F_{1} \equiv \frac{e \frac{d}{d \tau}\left[\left(a^{(1)} p\right)-\frac{e}{2}\left(a^{(1)}\right)^{2}\right]-e^{2} a^{(2)} \frac{d a^{(1)}}{d \tau}}{e\left[\left(a^{(1)}+a^{(2)}\right) p\right]-\frac{e^{2}}{2}\left(a^{(1)}+a^{(2)}\right)^{2}+\frac{\left(k^{(1)} p\right)\left(k^{(2)} p\right)}{k^{(1)} k^{(2)}}}, \\
& F_{2} \equiv \frac{e \frac{d}{d \tau}\left[\left(a^{(2)} p\right)-\frac{e}{2}\left(a^{(2)}\right)^{2}\right]-e^{2} a^{(1)} \frac{d a^{(2)}}{d \tau}}{e\left[\left(a^{(1)}+a^{(2)}\right) p\right]-\frac{e^{2}}{2}\left(a^{(1)}+a^{(2)}\right)^{2}+\frac{\left(k^{(1)} p\right)\left(k^{(2)} p\right)}{k^{(1)} k^{(2)}}} .
\end{aligned}
$$

Equation (6) for phases of the fields takes the form

$$
\begin{aligned}
& d \zeta_{1}=\frac{\left(k^{(1)} p\right)}{m} \exp \left(\int_{\tau_{i}}^{\tau} F_{2} d \tau\right) d \tau, \\
& d \zeta_{2}=\frac{\left(k^{(2)} p\right)}{m} \exp \left(\int_{\tau_{i}}^{\tau} F_{1} d \tau\right) d \tau .
\end{aligned}
$$

The obtained solution described by Eqs. (5), (10)-(12) is an exact one and satisfies the initial conditions at $\tau=$ $\tau_{i}$. Hence, according to the Cauchy theorem, Eq. (5) represents the only possible format to search for a solution for $\pi_{\mu}(\varepsilon, \vec{p})$. It is easy to see that the above solution, applied to the single plane wave, coincides with that obtained in $[14,15]$. Consider the case when only one wave exists, $a^{(2)}=0$. Then

$$
\begin{aligned}
f_{1} \rightarrow & \frac{1}{\left(k^{(1)} p\right)}\left[e\left(a^{(1)} p\right)-\frac{e^{2}}{2}\left(a^{(1)}\right)^{2}\right] \\
& -\left[e\left(a^{(1)} p\right)-\frac{e^{2}}{2}\left(a^{(1)}\right)^{2}\right], \quad F_{2} \rightarrow 0, f_{2} \rightarrow 0 ;
\end{aligned}
$$

$\pi_{\mu}(\tau)$, defined for this case by Eqs. (5) and (13), is equivalent to the solution for a single wave, obtained in $[14,15]$. In addition to that, Eq. (13) contains the constant term that depends upon the initial condition for the considered plane at the moment $\tau_{i}$ when $\pi_{\mu}=p_{\mu}$. This constant is equal to zero if the plane wave is turned on adiabatically at the moment of $\tau_{i} \rightarrow-\infty$.

The case when $a^{(1)}$ and $a^{(2)}$ are two components of a single wave with $k_{\mu}=k_{\mu}^{(1)}=k_{\mu}^{(2)}, a_{\mu}=a_{\mu}^{(1)}=a_{\mu}^{(2)}$ may serve as another possible test. Then, Eq. (5) for $\pi_{\mu}$ takes the form

$$
\pi_{\mu}(\tau)=p_{\mu}-e a_{\mu}+k_{\mu}\left(f_{1}+f_{2}\right) .
$$

In this case, the waves are collinear and $\left(k^{(1)} k^{(2)}\right) \rightarrow 0$. The expressions in Eqs. (10) and (11) become uncertain, and it is necessary to use asymptotic rules for their calculations. Denoting $\left(k^{(1)} k^{(2)}\right) \equiv x$ and considering the limits $\bar{F}_{1,2} \equiv \lim _{x \rightarrow 0} F_{1,2}$ and $\bar{f}_{1,2} \equiv \lim _{x \rightarrow 0} f_{1,2}$, we obtain

$$
\begin{aligned}
& \bar{f}_{1}=\frac{1}{(k p)}\left\{\left[e\left(a^{(1)} p\right)-\frac{e^{2}}{2}\left(a^{(1)}\right)^{2}\right]-e^{2} \int_{\tau_{i}}^{\tau} a^{(2)} \frac{d a^{(1)}}{d \tau} d \tau+c_{1}\right\}, \\
& \bar{f}_{2}=\frac{1}{(k p)}\left\{\left[e\left(a^{(2)} p\right)-\frac{e^{2}}{2}\left(a^{(2)}\right)^{2}\right]-e^{2} \int_{\tau_{i}}^{\tau} a^{(1)} \frac{d a^{(2)}}{d \tau} d \tau+c_{2}\right\}, \\
& \bar{f}_{1}+\bar{f}_{2}=f=\frac{1}{(k p)}\left[e\left(a^{(2)} p\right)-\frac{e^{2}}{2}\left(a^{(2)}\right)^{2}\right]+\text { const. }
\end{aligned}
$$

Then, $\pi_{\mu}$ defined by Eq. (14) duplicates again the solution for a single wave, except for the constant terms in Eq. (15). This solution can also be obtained immediately from the initial differential Eq. (9), assuming $\left(k^{(1)} k^{(2)}\right)=0$.

Hence, from the general solution for two arbitrary waves it is possible to arrive at the limit of the solution for a single plane wave obtained previously in $[14,15]$. It is tempting to look for a solution of Eq. (1) for more than two waves. However, the described approach does not permit us to find the exact solution for three waves, even for the case of the same linear polarization.

\section{PARTICLE IN PLANE-PARALLEL OPTICAL RESONATOR}

Let us apply Eqs. (5), (10), and (11) to the particular case of a standing EM wave formed by two linearly polarized plane waves of the same frequency $\omega$, counterpropagating along the $x$ axis and polarized along the $y$ axis with

$$
\begin{aligned}
a_{y}^{(1)}\left(\zeta_{1}\right) & =-\frac{E_{0}}{2 \omega} \cos \zeta_{1}, \quad \zeta_{1}=\omega(t-x), \\
a_{y}^{(2)}\left(\zeta_{2}\right) & =\frac{E_{0}}{2 \omega} \cos \zeta_{2}, \quad \zeta_{2}=\omega(t+x), \\
A_{y} & =a_{y}^{(1)}+a_{y}^{(2)}=-\frac{E_{0}}{\omega} \sin \omega t \sin \omega x,
\end{aligned}
$$

where $E_{0}$ is the amplitude of electric field. A standing wave is confined between two conducting surfaces (mirrors) placed at $x=0$ and $x=L=n \lambda / 2=n \pi / \omega, n=$ $1,2,3, \ldots$.

The electron moving along the axis of the optical resonator enters the cavity at the moment $t=t_{i}\left(\tau_{i}\right)$ and leaves it at $t=t_{f}\left(\tau_{f}\right)(t$ is time in the laboratory scale, $\tau$ is the local time of the moving electron). Time of flight 
in the lab system is

$$
T=t_{f}-t_{i}=\int_{\tau_{i}}^{\tau_{f}} \gamma d \tau=\frac{1}{m} \int_{\tau_{i}}^{\tau_{f}} \pi_{0} d \tau,
$$

where $\gamma$ is a Lorentz factor of the electron.

Let us consider a case of the weak field, when normalized field amplitude is $\eta^{2}=\frac{e^{2} a^{2}}{m^{2}} \ll 1$, where $a=\frac{E_{0}}{2 \omega}$ is the amplitude of the potentials in Eq. (16). In this case, Eq. (11) for $F_{1,2}$ can be expanded in series of $\eta^{2}$ and expressed as

$$
\begin{aligned}
& F_{1} \approx \frac{k^{(1)} k^{(2)}}{\left(k^{(1)} p\right)\left(k^{(2)} p\right)}\left\{e \frac{d}{d \tau}\left[\left(a^{(1)} p\right)-\frac{e}{2}\left(a^{(1)}\right)^{2}\right]-e^{2} a^{(2)} \frac{d a^{(1)}}{d \tau}\right\}, \\
& F_{1} \approx \frac{k^{(1)} k^{(2)}}{\left(k^{(1)} p\right)\left(k^{(2)} p\right)}\left\{e \frac{d}{d \tau}\left[\left(a^{(2)} p\right)-\frac{e}{2}\left(a^{(2)}\right)^{2}\right]-e^{2} a^{(1)} \frac{d a^{(2)}}{d \tau}\right\} ;
\end{aligned}
$$

subsequently, Eq. (10) for $f_{1,2}$ takes the form

$$
\begin{aligned}
& f_{1} \approx \frac{e}{\left(k^{(1)} p\right)}\left\{\left[\left(a^{(1)} p\right)-\frac{e}{2}\left(a^{(1)}\right)^{2}\right]-\left[\left(a^{(1)} p\right)-\frac{e}{2}\left(a^{(1)}\right)^{2}\right]_{i}-e \int_{\tau_{i}}^{\tau} a^{(2)} \frac{d a^{(1)}}{d \tau} d \tau\right\}, \\
& f_{2} \approx \frac{e}{\left(k^{(2)} p\right)}\left\{\left[\left(a^{(2)} p\right)-\frac{e}{2}\left(a^{(2)}\right)^{2}\right]-\left[\left(a^{(2)} p\right)-\frac{e}{2}\left(a^{(2)}\right)^{2}\right]_{i}-e \int_{\tau_{i}}^{\tau} a^{(1)} \frac{d a^{(2)}}{d \tau} d \tau\right\} .
\end{aligned}
$$

The expansion in Eq. (19) is valid for arbitrary orientation of the electron initial momentum $p_{\mu}$, in particular when $\vec{p}$ is normal to $\vec{k}^{(1,2)}$ or collinear with $\vec{k}^{(1)}$ or $\vec{k}^{(2)}$. In the ultrarelativistic case considered below, when $\vec{p}$ is collinear to $\vec{k}^{(1)}$, we obtain $k^{(1)} p=\omega(\varepsilon-p) \approx \omega \varepsilon \frac{m^{2}}{2 p^{2}}, k^{(2)} p=\omega(\varepsilon+p) \approx 2 \omega \varepsilon$, and $\left|f_{2}\right| \ll\left|f_{1}\right|$.

By Eq. (19),

$$
f_{1 f}=-\frac{2 p^{2} \eta^{2}}{\omega}\left\{\frac{1}{2}\left(\cos ^{2} \zeta_{1 f}-\cos ^{2} \zeta_{1 i}\right)-\int_{\zeta_{1 i}}^{\zeta_{1 f}} \cos \zeta_{2} \sin \zeta_{1} d \zeta_{1}\right\}
$$

where

$$
\zeta_{1 i}=\omega t_{i}\left(t=t_{i}, x=0\right), \quad \zeta_{1 f}=\omega\left(t_{i}+T\right)-\omega L,
$$

$L$ is the length of the resonator and $T$ is the time of flight, that by Eqs. (4), (5), and (17) are

$$
\begin{aligned}
T & \equiv t_{f}-t_{i}=\frac{1}{m} \int_{\tau_{i}}^{\tau_{f}} \pi_{0} d \tau=\frac{\varepsilon}{m}\left(\tau_{f}-\tau_{i}\right)+\frac{\omega}{m} \int_{\tau_{i}}^{\tau_{f}}\left(f_{1}+f_{2}\right) d \tau \approx \frac{\varepsilon}{m}\left(\tau_{f}-\tau_{i}\right)+\frac{\omega}{m} \int_{\tau_{i}}^{\tau_{f}} f_{1} d \tau, \\
L & =\frac{p}{m}\left(\tau_{f}-\tau_{i}\right)+\frac{\omega}{m} \int_{\tau_{i}}^{\tau_{f}}\left(f_{1}+f_{2}\right) d \tau \approx \frac{p}{m}\left(\tau_{f}-\tau_{i}\right)+\frac{\omega}{m} \int_{\tau_{i}}^{\tau_{f}} f_{1} d \tau .
\end{aligned}
$$

In order to estimate the integral entering Eq. (20) for $f_{1}$ in the weak field regime, it is possible to adopt the approximate expressions for $d \zeta_{1}$ and $d \zeta_{2}$ from Eq. (6),

$$
\begin{gathered}
d \zeta_{1} \approx \frac{k^{(1)} p}{m} d \tau, \quad \zeta_{1}=\frac{k^{(1)} p}{m}\left(\tau-\tau_{i}\right)+\zeta_{1 i}, \\
d \zeta_{2} \approx \frac{k^{(2)} p}{m} d \tau, \quad \zeta_{2}=\frac{k^{(2)} p}{m}\left(\tau-\tau_{i}\right)+\zeta_{2 i}, \quad \zeta_{2 i}=\zeta_{1 i}, \\
d \zeta_{2} \approx \frac{\left(k^{(2)} p\right)}{\left(k^{(1)} p\right)} d \zeta_{1}, \quad \zeta_{2}=\frac{k^{(2)} p}{k^{(1)} p}\left(\zeta_{1}-\zeta_{1 i}\right)+\zeta_{1 i} .
\end{gathered}
$$

Then it is not difficult to show that the integral in Eq. (20) is equal to the sum of terms which are, in the considered ultrarelativistic case, proportional to

Thus these terms can be neglected and

$$
\frac{\left(k^{(1)} p\right)}{\left(k^{(2)} \pm k^{(1)}, p\right)} \sim \frac{1}{4 \gamma^{2}}
$$

$$
f_{1 f}=-\frac{p^{2} \eta^{2}}{\omega}\left(\cos ^{2} \zeta_{1 f}-\cos ^{2} \zeta_{1 i}\right)
$$


Using Eqs. (23) and (24), it is possible to express $T$ through the local time interval $\left(\tau_{f}-\tau_{i}\right)$,

$$
T=\frac{\varepsilon}{m}\left(\tau_{f}-\tau_{i}\right)-\frac{p^{2} \eta^{2}}{m \varepsilon}\left\{\left(\frac{1}{2}-\cos ^{2} \zeta_{1 i}\right)+\frac{1}{4} \frac{m}{\left(k^{(1)} p\right)\left(\tau_{f}-\tau_{i}\right)}\left[\sin \left(\frac{2 k^{(1)} p}{m}\right)\left(\tau_{f}-\tau_{i}\right)+\sin 2 \zeta_{1 i}\right]\right\}\left(\tau_{f}-\tau_{i}\right),
$$

and to find a corresponding relation between $L$ and $\left(\tau_{f}-\tau_{i}\right)$.

For a short resonator

$$
\frac{k^{(1)} p\left(\tau_{f}-\tau_{i}\right)}{m} \approx \frac{\omega L}{2 \gamma^{2}} \ll 1, \quad(\omega L=n \pi) ;
$$

from Eqs. (22) and (25), it follows that

$$
\begin{gathered}
T=\frac{\varepsilon}{m}\left(\tau_{f}-\tau_{i}\right), \quad L=\frac{p}{m}\left(\tau_{f}-\tau_{i}\right), \\
T=\frac{\varepsilon}{p} L .
\end{gathered}
$$

For a long resonator

$$
\frac{k^{(1)} p\left(\tau_{f}-\tau_{i}\right)}{m} \approx \frac{\omega L}{2 \gamma^{2}} \gg 1,
$$

and

$$
\begin{aligned}
& T \approx \frac{\varepsilon}{m}\left[1-\frac{p^{2} \eta^{2}}{\varepsilon^{2}}\left(\frac{1}{2}-\cos ^{2} \zeta_{1 i}\right)\right]\left(\tau_{f}-\tau_{i}\right), \\
& L=\frac{p}{m}\left[1-\frac{p \eta^{2}}{\varepsilon}\left(\frac{1}{2}-\cos ^{2} \zeta_{1 i}\right)\right]\left(\tau_{f}-\tau_{i}\right),
\end{aligned}
$$

or

$$
T=\frac{\varepsilon}{p} L\left[1+\frac{m^{2} \eta^{2}}{2 p \varepsilon}\left(\frac{1}{2}-\cos ^{2} \zeta_{1 i}\right)\right] .
$$

Using Eqs. (21), (27), and (29), the final phase $\zeta_{1 f}$ is

$$
\zeta_{1 f} \approx \zeta_{1 i}+\omega L \frac{\varepsilon-p}{p} \approx \zeta_{1 i}+\frac{\omega L}{2 \gamma^{2}}
$$

Note that for an arbitrary $\tau\left(\tau_{i}<\tau<\tau_{f}\right)$ and the corresponding propagation length $l<L$,

$$
\zeta_{1}(\tau)=\zeta_{1 i}+\frac{\omega l}{2 \gamma^{2}}
$$

We can define the coherent interaction distance $d_{c}$, which corresponds to the distance where the phase change of the copropagating wave is $\Delta \zeta_{1}=\zeta_{1}-\zeta_{1 i}=\pi$ (compare with coherent radiation distance or radiation formation zone [18]). Then, according to Eq. (31a), $d_{c}=$ $\frac{2 \pi}{\omega} \gamma^{2}=\lambda \gamma^{2}$ and the definitions for short and long resonators in Eqs. (26) and (28) can be modified as follows: $L \ll d_{c}$ for the short resonator and $L \gg d_{c}$ for the long resonator. The distance $d_{c}$ strongly depends on $\gamma$. Thus it is possible to establish conditions according to Eqs. (26) and (28) just by varying the electron energy while the resonator length is fixed.

When trying to consider the strong field case $\eta^{2}=$ $\frac{e^{2} a^{2}}{m^{2}} \gg 1$, we realize that straightforward calculation of the integrals in Eqs. (10) and (11) is difficult due to rapid variations of the expressions under the integrals. An alternative approach that involves transformation and integration of general expressions for $f_{1,2}$, Eqs. (10) and (11), is necessary. Then we obtain

$$
\left[1+\frac{k^{(1)} k^{(2)}}{\left(k^{(2)} p\right)} f_{1}\right] \times\left[1+\frac{k^{(1)} k^{(2)}}{\left(k^{(1)} p\right)} f_{2}\right]=\frac{-e\left[\left(a^{(1)}+a_{1}^{(2)}\right) p\right]-\frac{e^{2}}{2}\left(a^{(1)}+a^{(2)}\right)^{2}+\frac{\left(k^{(1)} p\right)\left(k^{(2)} p\right)}{k^{(1)} k^{(2)}}}{e\left[\left(a^{(1)}+a_{1}^{(2)}\right) p\right]_{i}-\frac{e^{2}}{2}\left(a^{(1)}+a^{(2)}\right)_{i}^{2}+\frac{\left(k^{(1)} p\right)\left(k^{(2)} p\right)}{k^{(1)} k^{(2)}}}
$$

Equation (32) is exact, valid for arbitrary orientation of $\vec{p}$ and $\vec{k}^{1,2}$ and for an arbitrary field strength $\eta$.

For counterpropagating plane waves $k_{\mu}^{(1)}$ and $k_{\mu}^{(2)}$, linearly polarized along the $y$ axis, and with $\vec{p}$ directed along $k_{1}\left(\vec{k}_{x}, 0,0\right)$, Eq. (32) takes the form

$$
\left[1+\frac{k^{(1)} k^{(2)}}{\left(k^{(2)} p\right)} f_{1}\right] \times\left[1+\frac{k^{(1)} k^{(2)}}{\left(k^{(1)} p\right)} f_{2}\right]=\frac{\eta^{2}\left(\cos \zeta_{1}-\cos \zeta_{2}\right)^{2}+1}{\eta^{2}\left(\cos \zeta_{1}-\cos \zeta_{2}\right)_{i}^{2}+1}=1+\eta^{2} X^{2},
$$

where the condition $\zeta_{1 i}=\zeta_{2 i}$ is taken into account and

$$
X^{2} \equiv\left(\cos \zeta_{1}-\cos \zeta_{2}\right)^{2}
$$

Equation (33) can be rewritten as follows:

$$
\frac{2 \omega}{\varepsilon+p} f_{1}+\frac{2 \omega}{\varepsilon-p} f_{2}+\frac{4 \omega^{2}}{m^{2}} f_{1} f_{2}=\eta^{2} X^{2}
$$

Let us apply Eq. (35) first for small values $\eta^{2} \ll 1$ that correspond to $E_{0} \rightarrow 0$ (or $\omega \rightarrow \infty$ ). Take into account that $f_{1,2}$ are dimensionless and must be proportional to $\eta$, $E_{0}$, and $\omega$, in certain power. Then, it is evident that for $\eta^{2} \ll 1$ Eq. (35) can be satisfied when

$$
f_{1}=\frac{m^{2}}{\left(k^{(1)} p\right)} \eta^{2} y_{1}, \quad f_{2}=\frac{m^{2}}{\left(k^{(2)} p\right)} \eta^{2} y_{2},
$$

where $y_{1,2}$ are functions of $\zeta_{1,2}$ and $y_{1}+y_{2}=X^{2}$.

From Eq. (19), it is possible to find $y_{1,2}$ and to see that the condition $y_{1}+y_{2}=X^{2}$ is satisfied indeed. That means that the previously obtained approximate solution for $\eta^{2} \ll 1$, Eq. (19), fulfills the exact Eq. (35) up to the terms proportional to $\eta^{4}$. Notice that the interchange $k^{(1)} \leftrightarrow k^{(2)}$ in Eq. (36) provides the interchange $f_{1} \leftrightarrow f_{2}$ as it is supposed to be, based on physical reasons. 
For the strong field case $\eta^{2} \gg 1$, we use similar arguments as for $\eta^{2} \ll 1$ above. First let us notice that the condition $\eta^{2} \gg 1$ is fulfilled when $E_{0} \rightarrow \infty$ (or $\omega \rightarrow 0$ ). After applying the limit $\eta^{2} \rightarrow \infty$ to the left- and right-hand sides of Eq. (35), we see that only the last term in the left-hand side of Eq. (35) survives, and the solution for $f_{1,2}$ must be

$$
f_{1}=\frac{m^{2}}{2\left(k^{(1)} p\right)} \eta y_{1}, \quad f_{2}=\frac{m^{2}}{2\left(k^{(2)} p\right)} \eta y_{2},
$$

where $y_{1,2}$ are functions of $\zeta_{1,2}$ and $y_{1} y_{2}=X^{2}$. The simplest choice that provides the symmetry condition, $k^{(1)} \leftrightarrow k^{(2)}, f_{1} \leftrightarrow f_{2}$, is $y_{1}=y_{2}=|X|$ as is adopted below.

From Eqs. (6) and (37), with $y_{1}=y_{2}=|X|$ for $\eta^{2} \gg$ 1 , we have approximately

$$
\begin{gathered}
d \zeta_{1} \approx \eta|X| \frac{k^{(1)} p}{m} d \tau, \quad d \zeta_{2} \approx \eta|X| \frac{k^{(2)} p}{m} d \tau, \\
\frac{d \zeta_{2}}{d \zeta_{1}}=\frac{\left(k^{(2)} p\right)}{\left(k^{(1)} p\right)} \approx 4 \gamma^{2} \gg 1, \\
\zeta_{2}-\zeta_{2 i}=\frac{k^{(2)} p}{k^{(1)} p}\left(\zeta_{1}-\zeta_{1 i}\right) .
\end{gathered}
$$

Note that the exact general expression by Eq. (32) permits us to consider the strong field case for the electrons with the momentum perpendicular to the standing wave axis. Such geometry is used for experimental investigation of the Kapitza-Dirac effect [2] and in the Shintake beam profile monitor [6-9].

\section{PHASE SENSITIVE COMPTON SCATTERING IN STANDING EM WAVE}

The total radiated energy during the passage of the electron through the optical resonator of the length $L$ is

$$
\Delta E=\frac{2}{3} e^{2} \int_{t_{i}}^{t_{f}} w^{2} d t=\frac{2 e^{2}}{3 m} \int_{\tau_{i}}^{\tau_{f}} w^{2} \pi_{0} d \tau,
$$

where $w$ is the four-vector of acceleration

$$
w^{2}=\frac{1}{m^{2}} \frac{d \pi_{\mu}}{d \tau} \frac{d \pi_{\mu}}{d \tau} .
$$

For the weak field case $\eta^{2} \ll 1, \pi_{\mu}$ is given by Eqs. (5), (10), (11), (18), and (19) [for the strong field case, use Eq. (37) instead of Eqs. (18) and (19)].

Consider the ultrarelativistic electron moving along the standing wave axis in the weak field approximation. Then, the expression under integral in Eq. (39), up to the terms proportional to $\eta^{4}$, is

$$
\begin{aligned}
w^{2} \pi_{0} \approx \frac{e^{2} \varepsilon}{m^{4}} & \left\{\left(\frac{d a^{(2)}}{d \zeta_{2}}\right)^{2}\left(k^{(2)} p\right)+\left(\frac{d a^{(2)}}{d \zeta_{2}}\right)^{2} f_{1}\right. \\
& \left.\times\left[2\left(k^{(2)} p\right)\left(k^{(1)} k^{(2)}\right)+\frac{\omega}{\varepsilon}\left(k^{(2)} p\right)\right]\right\} .
\end{aligned}
$$

In Eq. (41), we use Eq. (6) for phases and an approximate expression

$$
\left(\frac{d \zeta_{2}}{d \tau}\right)^{2} \approx\left(\frac{k^{(2)} p}{m}\right)^{2}+2 \frac{\left(k^{(2)} p\right)\left(k^{(1)} k^{(2)}\right)}{m^{2}} f_{1},
$$

valid up to the terms proportional to $\eta^{2}$.

Estimating the integral in Eq. (39), using Eq. (41) up to the terms proportional to $\eta^{4}$ and of the order of $O\left(\gamma^{-1}\right)$, it is possible to use approximate expressions for phases $\zeta_{1}$ and $\zeta_{2}$ described by Eq. (23). Results of such calculations are slightly different for long cavities $L \omega / 2 \gamma^{2} \gg 1$ and short cavities $L \omega / 2 \gamma^{2} \ll 1$,

$\Delta E=\frac{1}{3} \frac{e^{2} \eta^{2} \omega^{2} \varepsilon(\varepsilon+p)^{2}}{m^{3}}\left(\tau_{f}-\tau_{i}\right)\left[1+3 \eta^{2} \varphi\left(\zeta_{1}\right)\right]$,

$$
\varphi\left(\zeta_{1 i}\right)= \begin{cases}\frac{1}{2}-\cos ^{2} \zeta_{1 i}, & \frac{L \omega}{2 \gamma^{2}} \gg 1, \\ \frac{1}{2}-\cos ^{2} \zeta_{1 i}+\cos \zeta_{1 i}, & \frac{L \omega}{2 \gamma^{2}} \ll 1 .\end{cases}
$$

Using Eqs. (27) and (30) for the time of flight $T$ through the short and long resonators, correspondingly, we obtain the following expressions for the average intensity of the scattered radiation $I=\frac{\Delta E}{T}$ : For a long resonator

$I_{\text {long }} \approx \frac{1}{3} \frac{e^{2} \eta^{2} \omega^{2}(\varepsilon+p)^{2}}{m^{2}}\left[1+4 \eta^{2}\left(\frac{1}{2}-\cos ^{2} \zeta_{1 i}\right)\right]$,

and for a short resonator

$$
\begin{aligned}
I_{\text {short }} \approx & \frac{1}{3} \frac{e^{2} \eta^{2} \omega^{2}(\varepsilon+p)^{2}}{m^{2}} \\
& \times\left[1+3 \eta^{2}\left(\frac{1}{2}-\cos ^{2} \zeta_{1 i}+\frac{1}{2} \cos \zeta_{1 i}\right)\right] .
\end{aligned}
$$

In the cm-gram-sec (CGS) system, the right-hand sides of Eqs. (42) and (43) need to be multiplied by the factor $c^{-7}$, the right-hand sides of Eqs. (44) and (45) need to be multiplied by the factor $c^{-5}$, and $p$ will be replaced by pc. Equations (44) and (45) comply, to the precision of up to $\eta^{2}$, with the results obtained for a single plane wave in Refs. $[14,15]$ (if we take $\vec{p}=0$ ). The only difference is due to the terms proportional to $\eta^{4}$ which depend upon the initial phase. The dependence of Eqs. (44) and (45) on phase can be used to control the intensity of the nonlinear Compton scattering, as in the case of the electron microbunch diagnostics. Terms proportional to $\eta^{4}$ disappear after averaging Eqs. (44) and (45) over the initial phase $\zeta_{1 i}$.

For the strong field case, when $\eta^{2} \gg 1$, it follows from Eqs. (5), (37), and (38) that the total radiated energy during the electron passage through the optical resonator is 


$$
\begin{aligned}
& \Delta E= \frac{2 e^{2}}{3 m} \int_{\tau_{i}}^{\tau_{j}} w^{2} \pi_{0} d \tau \\
&=\frac{2 e^{2} \varepsilon \eta^{2}}{3 m} \int_{\zeta_{1 i_{i}}}^{\zeta_{1 j}}(1+\eta|X|) \\
& \quad \times\left[\frac{\left(k^{(1)} p\right)}{m} \sin ^{2} \zeta_{1}+\frac{\left(k^{(2)} p\right)^{2}}{m\left(k^{(1)} p\right)} \sin ^{2} \zeta_{2}-2 \frac{\left(k^{(2)} p\right)}{m} \sin \zeta_{1} \sin \zeta_{2}-\frac{\left(k^{(1)} k^{(2)}\right) m}{\left(^{(1)} p\right)} \frac{d|X|}{d \zeta_{1}} \frac{d|X|}{d \zeta_{2}}\right]|X| d \zeta_{1} .
\end{aligned}
$$

For the ultrarelativistic case, the second term inside the square brackets in Eq. (46) provides the main contribution to the approximate expression for $\Delta E$ :

$$
\begin{aligned}
\Delta E & \approx \frac{16 \varepsilon^{4} \eta^{4} e^{2} \omega}{3 m^{4}} \int_{\zeta_{1 i_{i}}}^{\zeta_{1 j}} \sin ^{2} \zeta_{2}\left(\cos \zeta_{1}-\cos \zeta_{2}\right) d \zeta_{1} \approx \frac{8 \varepsilon^{4} \eta^{4} e^{2} \omega}{3 m^{4}} \int_{\zeta_{1 i_{i}}}^{\zeta_{1 j}}\left(\cos ^{2} \zeta_{1}+1 / 2\right) d \zeta_{1} \\
& =\frac{8 \varepsilon^{4} \eta^{4} e^{2} \omega}{3 m^{4}}\left[\left(\zeta_{1 f}-\zeta_{1 i}\right)+\frac{1}{4}\left(\sin 2 \zeta_{1 f}-\sin 2 \zeta_{1 i}\right)\right] .
\end{aligned}
$$

In Eq. (47), in order to provide a rough characterization for the dependence of the integral radiation upon $\eta$ and $\zeta_{1 i}$, the rapid variation of $\zeta_{2}$ [see Eq. (38)] is taken into account, and $\sin ^{2} \zeta_{2}$ and $\cos ^{2} \zeta_{2}$ are set equal to their average values (equal to $1 / 2$ ).

From Eqs. (5), (23), (37), and (38), it follows that

$$
\begin{aligned}
T & =t_{f}-t_{i} \approx \frac{e \varepsilon^{2}}{m} \eta\left(\tau_{f}-\tau_{i}\right)|\bar{X}|, \\
L & =\frac{p e^{2}}{m} \eta\left(\tau_{f}-\tau_{i}\right)|\bar{X}|, \\
T & =\frac{e \varepsilon^{2}}{p} L
\end{aligned}
$$

where $|\bar{X}|$ means an average value of $|X|$. Taking into account that by Eq. (48)

$$
\zeta_{1 f}-\zeta_{1 i} \equiv \omega(T-L)=\omega L\left(\frac{\varepsilon}{p}-1\right) \approx \frac{\omega L}{2 \gamma^{2}},
$$

the following expressions for the integral radiated energy can be obtained from Eq. (47). For a short resonator $\left(\omega L / 2 \gamma^{2} \ll 1\right)$

$$
\Delta E=\frac{4 \varepsilon^{2} \eta^{4} e^{2}}{3 m^{2}} \omega^{2} L\left[1+\frac{1}{2} \cos 2 \zeta_{1 i}\right],
$$

and for a long resonator $\left(\omega L / 2 \gamma^{2} \gg 1\right)$

$$
\Delta E \approx \frac{8 e^{2} \varepsilon^{2} \eta^{4} \omega^{2} L}{3 m^{2}} .
$$

In Eqs. (50) and (51), the only terms with highest power of $\eta$ are presented. In the CGS system, the right-hand side of Eqs. (50) and (51) will be multiplied by the factor $c^{-6}$.

\section{ACCELERATION OF CHARGED PARTICLES IN PLANE-PARALLEL OPTICAL RESONATOR}

Using general solutions obtained in Sec. III for the electron passing through the radiation filled plane-parallel cavity, we can address a problem of electron acceleration in vacuum. The momentum of the electron at the exit from the cavity is

$$
p_{f}=p_{i}+\omega f_{1 f}-\omega f_{2 f} \approx p_{i}+\omega f_{1 f} .
$$

From Eqs. (19) and (24) for weak field case $\eta \ll 1$,

$$
\begin{aligned}
p_{f} & =p_{i}-\frac{p^{2} \eta^{2}}{\varepsilon}\left(\cos ^{2} \zeta_{1 f}-\cos ^{2} \zeta_{1 i}\right) \\
& =p_{i}\left\{1-\frac{p \eta^{2}}{\varepsilon}\left[\cos ^{2}\left(\zeta_{1 i}+\frac{L \omega}{2 \gamma^{2}}\right)-\cos ^{2} \zeta_{1 i}\right]\right\} .
\end{aligned}
$$

For a short resonator, described by Eq. (26),

$$
p_{f}=p_{i}\left[1-\frac{p \eta^{2}}{\varepsilon}\left(\frac{1}{2}-\cos ^{2} \zeta_{1 i}-\sin 2 \zeta_{1 i}\right)\right],
$$

and for a long resonator, described by Eq. (28), after averaging over small changes in frequency, $\frac{\Delta \omega}{\omega} \ll$ $\frac{4 \gamma^{2}}{\omega L} \ll 1$

$$
p_{f}=p_{i}\left[1-\frac{p \eta^{2}}{\varepsilon}\left(\frac{1}{2}-\cos ^{2} \zeta_{1 i}\right)\right] .
$$

Thus, if $\cos ^{2} \zeta_{1 i}>\frac{1}{2}$, the acceleration takes place. For example, if $\zeta_{1 i}=\omega t_{i}=0$ and $\eta^{2}=0.25$, then, after passing the resonator, $p_{f}=1.12 p_{i}$.

Using $m$ appropriately displaced resonator cavities with $\zeta_{1 i}^{k}=\zeta_{i}+2 \pi k$, where $\zeta_{1 i}^{k}$ is the phase at the entrance into the $k$ th resonator, and pumped by the same laser, we obtain

$$
\begin{aligned}
p_{j} & =p_{i}\left[1-\frac{p \eta^{2}}{\varepsilon}\left(\frac{1}{2}-\cos ^{2} \zeta_{1 i}\right)\right]^{m} \\
& \approx p_{i}\left[1-\frac{p m \eta^{2}}{\varepsilon}\left(\frac{1}{2}-\cos ^{2} \zeta_{1 i}\right)\right] .
\end{aligned}
$$

For the above example, $p_{j} \approx 2 p_{i}$ at $m=8$. If we double the laser power, $\eta^{2}$ becomes equal to 0.5 and two cavities 
are enough for approximate doubling of the electron momentum.

For the $x$ component of the electron momentum in the strong field case $\eta^{2} \gg 1$, using Eq. (37) we obtain

$$
p_{x}=p_{i x}+\omega\left(f_{1}-f_{2}\right)=p_{i x}(1+\eta|X|) .
$$

At the back end of the resonator $x=L=\frac{\lambda}{2} n, n=$ $1,2,3, \ldots$, and $X=0$. Note that the maximum value of $|X|,|X|_{\max }=2$, is attained inside the resonator at points $x=l_{\max }=\left(\frac{1}{4}+m\right) \lambda$ and at the moments of time $t=t_{\max }=\left(\frac{1}{4}+m\right) \frac{2 \pi}{\omega}$, where $m=1,2,3, \ldots$ If we manage to extract the electrons at these points and moments of time, they will acquire the maximum momentum $p_{x} \approx 2 \eta p_{i x}$. As follows from Eq. (57), the electron momentum $p_{x}$ is independent upon the initial phase $\zeta_{1 i}$, but, through $|X|$, it depends on the field phases at the actual moments of time and the electron position inside the resonator [see Eq. (34)].

Acceleration of electrons, given by Eqs. (54), (55), and (57), is due to the nonlinear part of the Lorentz force, even in the case of strong fields, when the field strength $\eta$ enters linearly in Eq. (57). In this case $(\eta \gg 1)$ it is difficult to find $f_{1,2}$ from Eqs. (10) and (11) directly, due to the rapid variation of the function under the integral on the local time scale. From general solutions in Eqs. (10) and (11), it seems, at first glance, that $F_{1,2}$ and, consequently, $f_{1,2}$ will not depend on $\eta$ when $\eta \rightarrow \infty$. This is true only when the phase dependent parts of dominators in Eq. (11) are nonzero, but pass zero multiple times, due to the rapid and strong variations of phases $a^{(1,2)}$ in the strong field of a resonator. Thus, the result expressed by Eq. (57) is justified.

\section{DISCUSSION}

In the present work, following the classical approach developed in $[14,15]$ for a single planar EM wave, the general exact solutions for electron motion in the field of two planar EM waves with the same linear polarization (as well as for copropagating or counterpropagating waves with arbitrary polarization) are obtained. Detailed consideration is given to problems of propagation and radiation of the ultrarelativistic electrons, moving along the standing EM wave axis.

The total radiated energy due to Compton scattering is defined primarily by the wave counterpropagating to the direction of the electron momentum. This feature is physically understandable, if we take into account that the ultrarelativistic electron moves practically in phase with the copropagating component of the standing wave and experiences quickly oscillating force from the counterpropagating component. Thus, we can expect that the overall angular and spectral spread of the radiated photons shall generally obey distributions obtained in $[14,15,19,20]$ for Compton (or Thomson) backscattering of a single laser beam on the relativistic electron beam. However, more detailed analysis may be necessary, especially for angular and frequency distributions of the radiation intensity that depend on the initial phase.

The copropagating wave affects the Compton backscattering process through localization of the EM field inside the finite planar optical cavity, and, as a consequence, through the dependence of the nonlinear component in Compton scattering upon the standing wave phase at the moment when the electron enters the cavity. This difference from the single wave case becomes more pronounced even in the weak field case when terms proportional to $\eta^{2 m}(m \geq 2)$ become appreciable. Phase dependence of the nonlinear Compton scattering in a standing laser wave may be used for microbunch characterization (bunch duration, longitudinal charge distribution) as required in the advanced laser acceleration experiments, such as the STELLA experiment at the Brookhaven ATF [12].

Contrary to Compton scattering, electron acceleration is primarily due to the component of the standing wave collinear with the electron propagation. The effect of the second counterpropagating wave is dumped due to the phase averaging.

In the classical approach considered here, the acceleration of electrons is due to the ponderomotive force related to the linear and nonlinear parts of the Lorentz force $[10,16,17]$. The facts that the electron-laser interaction is localized within the finite optical cavity and the accelerating force is nonlinear to the laser field circumvent the Lawson-Woodward (LW) theorem [21], which otherwise forbids the residual electron energy gain from EM waves in vacuum. For a detailed discussion of the applicability of the LW theorem and examples of vacuum laser accelerator schemes see Ref. [10].

Dependence of the electron momentum at the exit of the optical resonator upon the initial phase of the standing EM wave may be used for electron (positron) beam diagnostics as well. The results for the time of flight, Eq. (29), and the momentum change, Eqs. (53) and (54), show also that an appreciable energy modulation sufficient for electron (positron) microbunching inside the optical cavity, or within the $\sim 1 \mathrm{~m}$ drift space after passing the cavity, is possible at $\eta<0.1$ when the initial electron energy is $E_{0}<50 \mathrm{MeV}$. Unfortunately, the laser intensity corresponding to such $\eta$ (not talking about higher fields desirable for using the optical cavities as the accelerator stages) exceeds the damage threshold for material optical mirrors. Another limitation to direct application of the obtained solutions to practical optical resonators is due to the fact that transition effects at the electron entrance and exit through the material boundaries are not taken into account. This problem needs additional consideration, for example, along the lines of Refs. [18,22,23]. The transition effect on the integral intensity of Compton scattering becomes negligible, however, for long enough resonators. 
It would be desirable to apply the obtained solutions for a more practical case of the standing wave produced within the waist of counterpropagating focused laser beams. We plan to explore this approach both analytically and computationally in the future.

Finally, let us point out that the exact solution for the electron motion in the field of standing wave, obtained in Sec. II, has a broader range of potential applications that are only partially addressed in the presented paper. It is related not just to further concentration on the acceleration and diagnostics of electron beams considered here, but may be applied to other physical problems where classical solution is valid, i.e., when the energy of the radiated photon is much smaller than the electron energy.

[1] P.L. Kapitza and P.A. M. Dirac, Proc. Cambridge Phys. Soc. 29, 297 (1993).

[2] M. V. Fedorov, in Electron in Strong Laser Field (Nauka, Moscow, 1991).

[3] R. Pantell, G. Soncini, and H.E. Puthoff, IEEE J. Quantum Electron. 4, 905 (1968).

[4] B. G. Bagrov, Yu. I. Klimenko, and V. R. Khalikov, Zh. Eksp. Teor. Fiz. 57, 922 (1969).

[5] V. V. Appolonov, Yu. L. Kalachev, A. M. Prokhorov, and M. V. Fedorov, Pis'ma Zh. Eksp. Teor. Fiz 46, 61 (1986).

[6] T. Shintake, Nucl. Instrum. Methods Phys. Res., Sect. A 311, 453 (1992).

[7] T. Shintake et al., KEK Report No. 94-129, 1994.

[8] M.S. Ross et al., in Proceedings of the XVIII International Linear Accelerator Conference, Geneva, Switzerland, 1996 (CERN, Geneva, 1996), Vol. 1, p. 308.

[9] T. Shintake, KEK Report No. 96-81, 1996.

[10] E. Esarey, P. Sprangle, and J. Krall, Phys. Rev. E 52, 5443 (1995).
[11] T.C. Marshall in Advanced Accelerator Concepts, edited by P. Schoessow, AIP Conf. Proc. No. 335 (AIP, New York, 1995), p. 261.

[12] I. V. Pogorelsky, A. van Steenbergen, J. C. Gallardo, V. Yakimenko, M. Babzien, K. P. Kusche, J. Skaritka, W. D. Kimura, D.C. Quimby, K.E. Robinson, S.C. Gottschalk, L. J. Pastwick, L. C. Steinhauer, D. B. Cline, Y. Liu, P. He, F. Camino, I. Ben-Zvi, R. B. Fiorito, D. W. Rule, R.H. Pantell, and J. Sandweiss, in Proceedings of the 1st JAERI-Kansai International Workshop on Ultrashort-Pulse Ultrahigh-Power Lasers and Simulation for Laser-Plasma Interactions, Kyoto, Japan, 1997 (Japan Atomic Energy Research Institute, Japan, 1998), p. 25.

[13] L. D. Landau and E. M. Lifshitz, in Classical Theory of Fields (Nauka, Moscow, 1973), Sec. 47.

[14] A. I. Nikishov and V. I. Ritus, Zh. Eksp. Teor. Fiz 46, 776 (1964); 46, 1768 (1964).

[15] V.I. Ritus, in Trudi FIAN (Nauka, Moscow, 1979), Chap. 2, Sec. 4.

[16] M. Feldman and R. Y. Chiao, Phys. Rev. A 4, 352 (1971).

[17] S. P. Kuo and M. C. Lee, J. Plasma Phys. 52, 339 (1994).

[18] B. M. Bolotovsky, in Trudi FIAN (Nauka, Moscow, 1982), p. 95 .

[19] E. Esarey, S. K. Ride, and P. Sprangle, Phys. Rev. E 48, 3003 (1993).

[20] S. K. Ride, E. Esarey, and M. Baine, Phys. Rev. E 52, 5425 (1995).

[21] P. J. Woodward, J. IEE 93, 1554 (1947); J. D. Lawson, IEEE Trans. Nucl. Sci. 26, 4217 (1979); R. B. Palmer, Part. Accel. 11, 81 (1980); R. B. Palmer, BNL Report No. 61317, 1994.

[22] B. M. Bolotovsky and G. V. Voskresensky, Usp. Fiz. Nauk 88, 209 (1966).

[23] L. C. Steinhauer, R. D. Romea, and W. D. Kimura, in Advanced Accelerator Concepts, edited by P. Schoessow, AIP Conf. Proc. No. 398 (AIP, New York, 1996), p. 673. 